\title{
Pancreatic ketoacidosis: ketonemia associated with acute pancreatitis
}

\author{
Udaya M Kabadi
}

\begin{abstract}
Summary
Ketonaemia is well documented as a consequence of prolonged starvation, acute alcoholism, and uncontrolled diabetes mellitus. However, its occurrence in acute pancreatitis has not been described. In this report, three patients who manifested ketoacidosis at the time of presentation of acute pancreatitis are described. In none of these patients could ketoacidosis be attributed to any of the well known pathogenetic factors such as ethanol, diabetes mellitus or prolonged starvation. In one patient, both the serum ketone titres and increased anion gap persisted for several days during the recovery period, despite appropriate therapy (including restriction of oral intake or nasogastric suction, intravenous fluids, and analgesic administration), before declining in parallel with a decrease in serum lipase levels, and became undetectable following near normalisation of serum lipase. Therefore, we believe that pancreatic ketosis or ketoacidosis may be a distinct syndrome with ketogenesis being promoted and maintained by extremely high circulating pancreatic lipase concentrations.
\end{abstract}

Keywords: acute pancreatitis, ketoacidosis, pancreatic lipase

\section{Introduction}

Ketonuria and/or ketonaemia with or without acidosis are known to occur during prolonged starvation (starvation ketosis), with uncontrolled diabetes mellitus, usually of the insulindependent type (diabetic ketoacidosis), or following ethanol ingestion (alcoholic ketoacidosis). ${ }^{1-15}$ I describe herein three subjects who manifested ketoacidosis in association with acute pancreatitis. In all of them, acute pancreatitis was the initial diagnosis and ketoacidosis was detected on evaluation of serum chemistry profiles and arterial blood gas analyses determined on presentation. In two patients, acute pancreatitis was secondary to type $\mathrm{V}$ hyperlipidaemia while in the third it occurred following endoscopic retograde cholangiopancreatography. Thus, none of the known causes of ketonaemia described above were responsible for ketoacidosis in these patients. Markedly increased circulating lipase concentration secondary to acute pancreatitis may be the causative factor in promoting and sustaining ketoacidosis in these subjects.

\section{Methods}

During 1989, 20 subjects were hospitalised for management of acute pancreatitis; in 17 of these ketonaemia and/or ketonuria were detected at the time of admission. Fourteen of these were excluded from further evaluation because of their history of either diabetes mellitus (seven subjects), acute alcohol ingestion, and/or duration of symptomatology of over 72 hours, in order to eliminate the known pathogenetic factors promoting ketogenesis, ie, diabetic ketoacidosis, alcoholic ketoacidosis and starvation ketosis. The remaining three patients are described below.

All laboratory tests were determined by the clinical laboratory at the medical centre. Serum C-peptide and beta hydroxybutyrate concentrations in Patient 2 were assessed by a commercial laboratory (Metwest Clinical Lab, Phoenix, Arizona).

\section{Patient 1}

A 53-year-old white man with a diagnosis of pancreas divisum established two months earlier with endoscopic retrograde cholangiopancreatography during hospitalisation for an episode of acute pancreatitis, was readmitted for placement of a stent in the minor papilla into the duct of Santorini. He also had a history of alcoholism for several years. He developed diffuse abdominal and back pain a day after the placement of the stent. Pain became progressively more severe and eventually became intolerable with onset of incessant nausea and vomiting over the next day. At this time, physical examination documented the presence of severe respiratory distress with hyperventilation. Pulse was rapid and weak with blood pressure $100 / 60 \mathrm{mmHg}$ and normal body temperature, $97^{\circ} \mathrm{F}$. Abdominal examination revealed diffuse tenderness, both direct and rebound, as well as marked guarding and rigidity. Bowel sounds were absent. Rectal and other systemic examinations were within normal limits. Laboratory investigations are given in table 1. Laboratory data confirming the diagnosis of acute pancreatitis as well as 
Table 1 Laboratory investigations

\begin{tabular}{llll}
\hline & Patient 1 & Patient 2 & Patient 3 \\
\hline Haemoglobin $(\mathrm{g} / \mathrm{dl})$ & 11.5 & 6.7 & 11.0 \\
Leucocytes $\left(\times 10^{9} / \mathrm{l}\right)$ & 19.6 & 12.3 & 15.6 \\
\% neutrophils & 85 & 76 & 77 \\
Ketonuria & ++ & ++ & ++ \\
Glycosuria & - & - & - \\
Liver enzymes & mildly elevated & normal & normal \\
Alkaline phosphatase $(\mathrm{IU} / \mathrm{ml})$ & 175 & normal & normal \\
Bilirubin concentration & slight increase & normal & normal \\
Coagulation & normal & normal & normal \\
Serum cholesterol $(\mathrm{mM} / \mathrm{l})$ & 14.5 & 10.4 & 16.1 \\
Triglycerides $(\mathrm{mM} / \mathrm{l})$ & 2.6 & 45.0 & 52.2 \\
Serum lactate & normal & normal & normal \\
\hline
\end{tabular}

++ marked; - not present.

ketoacidosis are summarised in table 2. This patient was managed with appropriate manoeuvers, ie, nasogastric suction, intravenous fluids ( $5 \%$ dextrose in normal saline), antibiotics, and presurgical preparation in anticipation of exploratory laparotomy. However, he developed a generalised seizure, tachycardia and cardiac arrest and expired within two hours despite resuscitative measures. Autopsy confirmed the diagnosis of acute haemorrhagic pancreatitis, generalised peritonitis without a perforation of any viscus, and omental fat necrosis.

\section{Patient 2}

A 40-year-old white man with a history of paraplegia secondary to a spinal cord injury at 9 th and 10th thoracic spinal level, following a motor vehicle accident several years earlier was recovering during hospitalisation for a lobar pneumonia and being treated with appropriate antibiotics. The patient was also receiving gemfibrozil $600 \mathrm{mg}$ twice daily for treatment of type $\mathrm{V}$ hyperlipidaemia diagnosed six years earlier during hospitalisation for acute pancreatitis. On the 8th day of admission, the patient complained of severe respiratory distress over a few hours. On inquiry, the patient related mild abdominal pain with radiation to his back and nausea for two days. On review of the daily records, the patient had not fully consumed his meals during this period.

Physical examination revealed rapid pulse $120 / \mathrm{min}$; blood pressure $130 / 75 \mathrm{mmHg}$ in

Table 2 Summary of the pertinent laboratory data in three patients who presented with ketoacidosis in association with acute pancreatitis

\begin{tabular}{|c|c|c|c|c|}
\hline Tests & Patient 1 & Patient 2 & Patient 3 & Normal range \\
\hline Glucose $(\mathrm{mM} / \mathrm{l})$ & 4.8 & $2.8^{\star \star}$ & 8.0 & $3.4-6.4$ \\
\hline Urea $\mathbf{N}(\mathrm{mM} / \mathbf{l})$ & 13.3 & 20.1 & 6.8 & $2.9-7.8$ \\
\hline $\mathrm{HCO}_{3}(\mathrm{mM} / \mathrm{l})$ & 13 & 4 & 9 & $24-28$ \\
\hline Anion gap ${ }^{\star}(\mathrm{mM} / \mathrm{l})$ & 27 & $37 \dagger$ & 20 & $9-15$ \\
\hline Ketone (titres) & $1: 16$ & $1: 32$ & $1: 8$ & Neg \\
\hline $\mathrm{Ca}^{++}(\mathrm{mM} / \mathrm{l})$ & 2.51 & 1.80 & 2.00 & $2.15-2.62$ \\
\hline pH & 7.30 & 6.84 & 7.18 & $7.35-7.45$ \\
\hline $\mathrm{PCO}_{2}(\mathrm{mmHg})$ & 26 & 10 & 18 & $35-45$ \\
\hline Amylase $(\mathrm{U} / \mathrm{l})$ & 1740 & 2470 & 2101 & $25-115$ \\
\hline Lipase $(\mathbf{U} / \mathbf{l})$ & 428 & 1180 & 750 & $0-195$ \\
\hline
\end{tabular}

$\star$ Calculated as $\mathrm{Na}^{+}\left(\mathrm{Cl}^{-}+\mathrm{HCO}^{-}{ }_{3}\right)$

$\star \star$ Serum C-peptide was $2.5 \mathrm{nM} / 1$ (normal range $0.16-1.0 \mathrm{nM} / \mathrm{l}$ )

†Beta hydroxybutyrate in this patient was $2418 \mu \mathrm{M} / 1$ (normal range $0-300 \mu \mathrm{M} / \mathrm{l}$ ). supine position and $100 / 60 \mathrm{mmHg}$ while sitting; temperature $97^{\circ} \mathrm{F}$ and deep, rapid respirations, $32 / \mathrm{min}$. Clusters of eruptive xanthomata were noted on the back, thighs and buttocks. Mucus membranes appeared unremarkable. Mild tenderness, and decreased peristaltic sounds were noted on abdominal examination, although no guarding, rigidity or rebound tenderness were elicited. Chest examination confirmed tachypnoea, but no adventitious sounds. Rectal and other systemic examinations were unremarkable.

Pertinent laboratory data confirmed the diagnosis of both acute pancreatitis and ketoacidosis (table 2). Serum C-peptide concentration was evaluated with simultaneous hypoglycaemia at the time of diagnosis. Other significant laboratory data are given in table 1. The findings on abdominal CT scan appeared to be consistent with diagnosis of acute pancreatitis, with edematous body of pancreas.

The patient made a full recovery within the next week with intravenous administration of initial bolus of $50 \mathrm{ml}$ of $50 \%$ dextrose followed by liberal quantities of $5 \%$ dextrose along with insulin, withholding of oral intake until return of active bowel sounds followed by gradual progression of oral feeding initially with liquids and later with solids. The patient was discharged on the 17th day of hospitalisation with instructions to continue therapy with gemfibrozil. The patient's blood pressure continued to show moderate orthostatic change throughout hospitalisation.

\section{Patient 3}

A 41-year-old, obese, white man was admitted with a history of worsening abdominal pain radiating to the back accompanied by severe nausea, retching and recurrent vomiting of two days' duration. The patient reported a previous episode of similar illness requiring hospitalisation for several days and diagnosed to be acute pancreatitis approximately eight years ago. The major cause of this previous episode of pancreatitis was thought to be familial type $V$ hyperlipidaemia and hence the treatment with gemfibrozil was initiated. The patient had continued gemfibrozil for three years but had discontinued therapy during the five years prior to this hospitalisation. The patient was taking no medication at the time of admission. Family history revealed the presence of type $V$ hyperlipidaemia and recurrent acute pancreatitis and diabetes mellitus in his father.

Physical examination revealed: pulse 110/ min; temperature $99^{\circ} \mathrm{F}$; and blood pressure $130 / 90 \mathrm{mmHg}$ in supine position and 105/ $80 \mathrm{mmHg}$ while standing. The respirations were deep at a rate of $26 / \mathrm{min}$. Skin and mucous membranes were dry. Several eruptive xanthomata were present on chest, thighs and buttocks. Abdominal examination noted tenderness and guarding in the epigastric area, but no rigidity or rebound tenderness. Bowel sounds were hypoactive and distant. Rectal examination was normal and stool was guaiac negative. The rest of the systemic examination was normal with the exception of tachycardia.

The results of laboratory findings are given 
Table 3 Repeated determinations of serum lipase, arterial $\mathrm{pH}$, serum ketone titres and anion gap during treatment and follow-up over two weeks in patient 3. Normal figures are given in parentheses

\begin{tabular}{lllll}
\hline Day & $\begin{array}{l}\text { Lipase } \\
(0-195 \mu / l)\end{array}$ & $\begin{array}{l}p H \\
(7.35-7.45)\end{array}$ & $\begin{array}{l}\text { Serum ketone } \\
(\text { Neg })\end{array}$ & $\begin{array}{l}\text { Anion gap } \\
(9-15 \mathrm{mM} / \mathrm{l})\end{array}$ \\
\hline 1 & 750 & 7.33 & $1: 8$ & 20 \\
2 & 850 & 7.18 & $1: 16$ & 25 \\
3 & 710 & 7.32 & $1: 8$ & 23 \\
4 & 640 & 7.43 & $1: 4$ & 23 \\
5 & 500 & - & $1: 4$ & 17 \\
6 & 390 & - & $1: 4$ & 16 \\
9 & 410 & 7.43 & $1: 4$ & 18 \\
12 & 380 & - & $1: 2$ & 16 \\
15 & 330 & 7.41 & Neg & 12 \\
\hline
\end{tabular}

in tables 1 and 2. The diagnosis of acute pancreatitis was confirmed by abdominal CT scan.

The patient was managed with therapeutic measures, ie, analgesia, nasogastric suction, antacids, and intravenous fluids (5\% dextrose in normal saline) until resumption of oral intake. Serum amylase decreased gradually and normalised over one week. However, both serum ketone titers and anion gap levels remained elevated for almost two weeks despite a gradual decline after initial worsening, and normalised only with near normalisation of serum lipase concentration (table 3 ).

\section{Discussion}

Ketoacidosis in our patients cannot be attributed to a lack of oral intake, since ketonaemia and/or ketoacidosis is not a frequent manifestation of starvation. ${ }^{1,2}$ Furthermore, starvation could not be responsible for the persistence of ketonaemia in patient 2 , since abundant quantities of glucose were infused initially followed by resumption of oral intake during the period of observation. Alternatively, ketoacidosis is frequently attributed to ethanol ingestion. However, alcoholic ketoacidosis is unlikely since serum ketone titers are negative or only minimally elevated in this syndrome, ${ }^{6-9}$ whereas all our patients manifested moderate to severe ketonaemia and ketonuria. Furthermore, in none of these patients was alcohol an offending agent in precipitating acute pancreatitis. Another well-established pathogenetic factor in promoting ketoacidosis, namely diabetes mellitus ${ }^{3,4}$ was not present in any of our patients. Therefore, ketoacidosis in these subjects with acute pancreatitis could not be attributed to known causes (see box). Finally, markedly elevated levels of glucose counterregulatory hormones ie, catecholamine, cortisol, growth hormone, etc, noted in acute pancreatitis ${ }^{16-20}$ may contribute slightly to ketogenesis. However, ketonaemia is unlikely in the presence of adequate circulating insulin

1 Cahill GF, Jr. The physiology of insulin in man. Diabetes 1971; 20: 785-99.

2 Fery F, Balasse EO. Ketone body turnover during and after exercise in overnight-fasted and starved humans. $A m \mathcal{F}$ Physiol 1983; 245: E318-E325.

3 Kreisberg RA. Diabetic ketoacidosis: new concepts and trends in pathogenesis and treatment. Ann Intern Med 1978;
88: 681-95. levels since even minimal circulating insulin concentrations are known to blunt the effect of these hormones, as documented in hyperglycaemic hyperosmolar nonketotic states. ${ }^{21-24}$ Thus, ketonaemia is unlikely to be secondary to elevated glucose counter-regulatory hormones and/or insulin deficiency in our patients as circulatory insulin concentrations were adequate (as reflected by an increased serum C-peptide concentration in one subject and $\stackrel{\curvearrowright}{\&}$ lack of hyperglycaemia in the other two sub- $\subseteq$ jects on admission). Hypoglycaemia in patient $2 \stackrel{\vec{F}}{\stackrel{F}{*}}$ cannot be attributed to prolonged starvation as reflected by the adequate food intake during hospitalisation, as well as the increased C- $\overline{\bar{c}}$ peptide concentration. The enhanced endo- $\mathbb{D}$ genous insulin secretion in these patients may be secondary to its stimulation by markedly elevated free fatty acids and their products as described previously in studies assessing the $\overrightarrow{\vec{\omega}}$ effects of lipids on pancreatic $\alpha$ - and $\beta$-cell function. ${ }^{25,26}$ Therefore, it seems likely that the $\vec{\theta}$ acute pancreatitis itself was responsible for ketoacidosis in all these cases, since both ketonaemia and ketoacidosis resolved with recovery from the disease.

Pancreatic lipase is known to promote local $\stackrel{\omega}{N}$ fat digestion and necrosis following its leakage 음 into the mesentery, frequently resulting in hypocalcaemia. Two of the three patients manifested hypocalcaemia, thus suggesting ongoing mesenteric fat breakdown, while the $\stackrel{9}{\frac{9}{2}}$ third patient demonstrated mesenteric fat necrosis at autopsy. Therefore, ketoacidosis in these patients could be attributed to enhanced mobilisation of tissue adipose stores induced by elevated circulating lipase concentrations. This hypothesis is supported by our finding that, in one patient, serum ketone titers and anion gap increased initially despite institution of appropriate therapy before gradually declining over several days with a concurrent fall in serum lipase levels and became normal only following near normalisation of serum lipase concentration (table 3). It is also apparent that lipid breakdown induced by circulating pancreatic lipase may not be inhibited by insulin since ketoacidosis occurred despite adequate cir- $\delta$ culating insulin concentration as reflected by a raised serum C-peptide level in patient 2 who $ᄋ$ manifested hypoglycaemia and in the others with euglycaemia in conjunction with ketoacidosis. Therefore, we believe that ketosis or $N$ ketoacidosis secondary to acute pancreatitis is a distinct, though previously unrecognised syn- $N$ drome, induced and maintained by extremely $\omega$ high levels of pancreatic lipase in the circulation.

The authors thank Marcia Gregory and Vivian Lavendar for their secretarial assistance.

4 Foster DW, McGarry JD. The metabolic derangements and reatment of diabetic ketoacidosis. N Engl f Med 1983; 309: 159-69.

5 Jenkins DW, Eckel RE, Craig JW. Alcoholic ketoacidosis. JAMA 1971; 217: 177-83.

6 Levy LJ, Duga J, Girgis M, Gordan EE. Ketoacidosis associated with alcoholism in nondiabetic subjects. Ann Intern Med 1973; 78: 213-9. 
7 Fulop M, Hoberman HD. Alcoholic ketosis. Diabetes 1975; 24: 785-90.

8 Cooperman MT, Davidoff F, Spark R, Pallotta J. Clinical studies of alcoholic ketoacidosis. Diabetes 1971; 23: 433-9. 9 Miller PD, Henig RE, Waterhouse C. Treatment of alcoholic ketoacidosis. Arch Intern Med 1978; 138: 67-72.

10 Palmer JP. Alcoholic ketoacidosis: clinical and laboratory presentation, pathophysiology and treatment. Clin presentation, pathophysiology

11 Halperin ML, Hammeke M, Josse RG, Jungas RL. Metabolic acidosis in the alcoholic: a pathophysiological approach. Metabolism 1983; 32: 308-15.

12 Fulop M, Ben-Ezra J, Bock JL. Alcoholism. Clin Exp Res 1986; 10: 610-5.

13 Duffens K, Mark SA. Alcoholic ketoacidosis-a review. $f$ Emerg Med 1987; 5: 399-406.

14 Fulop M. Alcoholism, ketoacidosis and latic acidosis. Diabetes Metab Rev 1989; 5: 365-78.

15 Wrenn KD, Slovis CM, Minion GE, Rutkowski R. The syndrome of alcoholic ketoacidosis. Am $\mathcal{F}$ Med 1991; 91 119-28.

16 Drew SL, Joffe B, Vinik A, Seftel H, Singer F. The first 24 hours of acute pancreatitis: changes in biochemical and endocrine homeostasis in patients with pancreatitis endocrine homeostasis in patients with pancreatitis for reasons other than pancreatitis. Am $7 \mathrm{Med} 1978 ; 64$ : for reaso
17 Rayfield EJ, Curnow RT, George DT, Beisel ER. Impaired carbohydrate metabolism during a mild viral illness. $N$ Engl f Med 1973; 289: 618-21.

18 Rocha DM, Santevsanio F, Faloona GR, Unger $R$ Abnormal pancreatic alpha-cell function in bacterial infections. N Engl f Med 1973; 288: 700-3.

19 George DT, Rayfield EJ, Wannemacher RW. Altered George DT, Rayfield EJ, Wannemacher RW. Altered glucoregulatory hormones during acute pneumococcal

20 Rayfield EJ, Curnow RT, Reinhard D, Kochicheril NM Effects of acute endotoxemia on glucoregulation in normal Effects of acute endotoxemia on glucoregulation in normal
and diabetic subjects. $\mathcal{F}$ Clin Endocrinol Metab 1977; 45 513-2

21 Boyer MH. Hyperosmolar anacidotic coma in association with glucocorticoid therapy. $\mathcal{F} A M A$ 1967; 101: 1007-9.

22 Fulop M, Tannenbaum H, Dreyer N. Ketotic hyperosmolar coma. Lancet 1973; 2: 635-9.

23 Lindsey CA, Faloona GR, Unger RH. Plasma glucagon in nonketotic hyperosmolar coma. JAMA 1974; 229: 1771-3.

24 Gordon EE, Kabadi UM. The hyperglycemic hyperosmolar syndrome. Am f Med Sci 1976; 271: 252-68.

25 Madison LL, Seyffert WA Jr, Unger RH, Barker B. Effect of plasma free fatty acids on plasma glucagon and serum insulin plasma free fatty acids on plasma glucagon and

26 Andrews SS, Lopez AS, Blackard WG. Effect of lipids on glucagon secretion in man. Metabolism 1975; 24: 35-44. 